\title{
Civilisations
}

Revue internationale d'anthropologie et de sciences

humaines

$66 \mid 2017$

L'alcool rituel et les ethnographes

\section{Emplir les corps des dieux pour rassasier les}

hommes

Etude de manipulations rituelles de bière de sorgho (pays kabyè, Togo)

\section{Marie Daugey}

\section{(2) OpenEdition}

\section{Journals}

Édition électronique

URL : http://journals.openedition.org/civilisations/4287

DOI : $10.4000 /$ civilisations.4287

ISSN : 2032-0442

Éditeur

Institut de sociologie de l'Université Libre de Bruxelles

\section{Édition imprimée}

Date de publication : 31 août 2017

Pagination : 59-75

ISBN : 978-2-9602017-1-0

ISSN : 0009-8140

Référence électronique

Marie Daugey, "Emplir les corps des dieux pour rassasier les hommes », Civilisations [En ligne], 66 |

2017, mis en ligne le 31 août 2020, consulté le 25 février 2021. URL : http://journals.openedition.org/ civilisations/4287 ; DOI : https://doi.org/10.4000/civilisations.4287 


\title{
Emplir les corps des dieux pour rassasier les hommes
}

\author{
Etude de manipulations rituelles de bière de sorgho (pays kabyè, Togo)
}

\author{
Marie DAUGEY
}

Résumé : Chaque année, chez les Kabyè du Togo, le parcours rituel organisé pour soutenir le développement final du petit mil et annoncer la tenue de nouvelles sessions initiatiques donne lieu à une importante alcoolisation d'un jeune garçon, dont la participation au rite consiste avant tout à marcher, puis à boire. Sur quels ressorts symboliques l'efficacité de cette pratique rituelle s'appuie-t-elle? Après une description analytique et contextualisée de cet usage du boire, nous trouverons des éléments de réponse dans les représentations relatives au corps des enfants, et dans une mise en regard avec d'autres rituels qui impliquent d'emplir de bière ou de substances dérivées de la fabrication de la bière des réceptacles de forme humaine. Il s'agit dans tous les cas de créer l'image d'une réplétion agissant comme une injonction à l'abondance adressée aux divinités.

Mots-clés : bière de sorgho, rituel, Kabyè, Togo, corps, enfant.

\begin{abstract}
Each year, in the Kabye society, a boy takes part in a ritual route supporting the final growth of millet and announcing the beginning of initiations. Above all, his participation consists in walking and ingesting a large amount of sorghum beer. On which symbolical motives does the efficacy of this alcoholization practice rest? After describing and analyzing the context surrounding this drinking custom, some answers will arise from representations related to the children's body. This practice will also be put into perspective with other rites entailing to fill anthropomorphous receptacles with beer or with matters derived from beer making. All these rites aim to create the image of satiety, acting as a request for abundance addressed to the divinities.
\end{abstract}

Keywords: sorghum-beer, ritual, Kabye, Togo, body, child. 
Chez les Kabyè du Togo ${ }^{1}$, la bière de sorgho (cukudu ou sulum ${ }^{2}$ ) occupe une place importante dans la plupart des domaines de la vie sociale et religieuse. La consommation et/ou la manipulation de cette boisson est indispensable lors des événements qui marquent les grandes étapes de la vie individuelle et collective : les procédures d'alliance matrimoniale, de réconciliation, les rites initiatiques, les rites funéraires, les rites agraires et cynégétiques. Dans cette société, l'organisation des cycles rituels humains et agricoles reste étroitement liée à un culte des ancêtres, de type domestique et lignager, et à un culte des divinités du territoire, organisé à l'échelle d'une localité ou d'une subdivision territoriale et lignagère. La pratique de ces cultes implique le plus souvent de s'adresser à une entité en versant de la bière de sorgho sur son autel. Ces versements de bière permettent $d$ 'amorcer une situation de communication avec cette entité, en lui donnant à boire, et/ou en appuyant les paroles d'une prière. Ils sont couramment assortis de séquences de consommation de bière par les bénéficiaires du rite ou leurs représentants. Les pratiques de commensalité auxquelles donnent lieu ces cérémonies permettent de créer, renouveler ou modifier un lien entre les membres d'un groupe social (Fabre-Vassas 1989), ou entre un groupe humain (lignage, clan, village) et des divinités.

L'usage rituel de bière de sorgho, qui sera étudié plus précisément dans cet article, se distingue des manipulations rituelles ordinaires évoquées ci-dessus. Il est une forme intense de libation cérémonielle. Il consiste à faire boire une grande quantité de bière de sorgho à un enfant qui représente un « grand prêtre » (cojo), c'est-à-dire un desservant du culte aux divinités du territoire (ègolimiyè, pl. agolima). Cette pratique a lieu en un temps décisif du calendrier rituel annuel ${ }^{3}$. Elle accompagne le développement final du petit mil (mila, pl. misi) - premier aliment récolté dans l'année - et elle est intégrée à la procédure rituelle qui annonce le commencement prochain des initiations masculines et féminines.

Comment cette consommation de bière par un garçon peut-elle jouer un rôle important dans l'accompagnement d'événements majeurs de la vie sociale ? Sur quels ressorts symboliques l'efficacité de cette pratique rituelle s'appuie-t-elle ? Après une présentation des usages courants du boire en contexte quotidien et rituel, nous décrirons précisément le déroulement du rite de surconsommation de bière par l'enfant-prêtre. Notre réflexion prendra alors deux directions. Premièrement, nous interrogerons certaines représentations relatives aux spécificités attribuées au corps des enfants. Deuxièmement, nous mettrons en regard cette pratique singulière d'alcoolisation avec d'autres usages rituels qui impliquent eux aussi de créer l'image d'une réplétion en ayant recours à de la bière de sorgho ou à des matières dérivées de la fabrication de la bière.

1 Les Kabyè représentent le deuxième groupe ethnique du Togo par leur poids démographique. Les faits rapportés ici se déroulent chaque année dans ce que les Kabyè reconnaissent comme leur territoire d'origine. Situé au nord de la ville de Kara, celui-ci est composé de plusieurs massifs montagneux, et la population y est majoritairement agricultrice.

2 L'orthographe kabyè a été standardisée par le Comité de Langue Nationale Kabiyè en association avec la Société Internationale de Linguistique. Toutefois, pour faciliter la lecture, ce texte ne s'y conforme pas complètement. Les caractères spéciaux ont été latinisés.

3 Celui-ci organise l'articulation entre le cycle agricole et le cycle des initiations masculines et féminines, marquant le passage à l'âge adulte. 


\section{Usages quotidiens et rituels de la bière de sorgho}

\section{La bière au quotidien}

La bière de sorgho occupe une place capitale dans la vie quotidienne et les modes d'entretien des relations sociales. La préparation et la vente de la bière sont des tâches réservées aux femmes. Certaines en vendent une à deux fois par semaine, chez elles ou sur un marché, et y consacrent donc beaucoup de temps. ${ }^{4}$. Les principaux consommateurs de cette boisson sont les hommes, qui la boivent quasi-quotidiennement, après leur journée de travail, mais femmes et enfants en boivent aussi occasionnellement. Tout travail collectif de culture ou de construction, masculin ou féminin, s'achève par le partage d'un pot de bière fourni par le commanditaire de la séance de travail.

R. Verdier a souligné le rôle central de la bière dans l'animation d'un marché. «Un marché ne peut se concevoir sans vendeuse de bière »; " la bière fait asseoir le marché », écrit-il (1962 : 117). Ainsi est-il rare qu'un homme quitte un marché sans aller boire de la bière auprès de la vendeuse dont il apprécie la boisson ou la beauté. Boire et passer un moment agréable à discuter avec les autres consommateurs peut être l'unique raison pour laquelle se rendre dans un marché. Les discussions menées « sous les pots de cukudu » sont d'importants lieux d'échanges concernant les problèmes et événements survenus dans le village, le pays et le monde.

En plus de favoriser l'entretien des liens sociaux par le partage d'instants de convivialité ou de controverses, sur le plan symbolique, une vertu importante de cette boisson est sa capacité à régénérer la force de ses consommateurs. C. Piot (1999: 82) a montré que boire de la bière de sorgho est vu comme contribuant à construire la virilité d'un garçon en rendant son sang « fort », d'une manière comparable à la consommation du sang de chien pendant l'initiation masculine. Un homme peut ainsi affirmer que boire du cukudu lui fait « bouillir le sang ${ }^{5} »$. Seule une boisson bien fermentée, un peu amère, est considérée comme convenant à l'organisme masculin ; elle permettrait de le fortifier. La boisson non fermentée, sucrée, est dépréciée par les hommes (cela les affaiblirait) et considérée comme convenant davantage aux femmes et aux enfants.

La bière de sorgho est aussi un aliment qui rassasie. Une personne pauvre se contente parfois d'une calebasse de bière pour seul repas. Cette conception d'une boisson qui nourrit et fortifie est parfois exprimée en des termes qui vantent la valeur nutritionnelle du sorgho ${ }^{6}$.

Au quotidien, il est plutôt rare que la consommation de bière soit tournée vers une recherche de grande ivresse, sauf exceptions. La faible maîtrise de soi, voire la

4 La fabrication de cette boisson, déjà décrite ailleurs (Cridel 1968: 69 ; Verdier 1982 : 195 ; Roberts 2013 : 250-251) s'étale sur six jours. Le premier jour, le grain est immergé pour favoriser sa germination. Celle-ci exigera plusieurs manipulations pendant deux à trois jours (le grain est étalé, recouvert, rincé). Le cinquième jour, le grain germé est broyé puis déposé dans une jarre, recouvert d'eau et malaxé. Plusieurs opérations de décantation et de filtrage, ainsi que deux cuissons sont alors nécessaires pour obtenir le breuvage désiré.

5 Le caractère bouillonnant et rouge de la bière fermentée est également associé chez les Dogon à la catégorie du chaud et à « la force violente » (Jolly 2004 : 182).

6 C'est ainsi que m'en ont parlé notamment des étudiants loméens. 
violence à laquelle l'ébriété peut mener sont désapprouvées ${ }^{7}$. Les excès alcooliques, plus fréquemment masculins, se font souvent à l'aide d'alcools plus forts que la bière de sorgho locale, et associés au monde extérieur. Les « concours de boisson » auxquels s'adonnent parfois les jeunes cultivateurs sont l'exemple d'une pratique d'alcoolisation hors norme dépréciée par les générations précédentes. Ils se produisent après la vente des récoltes de tomates et de piments, qui est bien rémunératrice. De la bière et des alcools forts, manufacturés, sont bus pendant ces confrontations. Plus coûteux que la bière traditionnelle, ils sont associés à la modernité et à la richesse. Le sodabi est aussi consommé en ces occasions. Issu de la distillation du vin de palme et produit principalement dans le sud du Togo, il est souvent l'alcool privilégié pour atteindre rapidement l'ivresse ${ }^{8}$. Les personnes qui évoquent les jeunes hommes ayant frôlé ou trouvé la mort dans ces concours insistent sur les quantités impressionnantes d'alcool qui avaient été bues, et déclenchent la stupéfaction de leurs interlocuteurs.

D'ordinaire, l'ivresse féminine est plutôt désapprouvée. Une femme qui, comme les hommes, se rendrait en fin de journée auprès d'une vendeuse de bière pour boire et bavarder serait vue comme négligeant ses obligations domestiques. "Une femme ne peut pas boire comme le font les hommes. Elle doit s'occuper des enfants à la maison, préparer le repas du soir, elle n'a pas le temps pour ça !", indiqua par exemple un interlocuteur masculin. Les femmes boivent davantage lors de cérémonies festives où la bière est présente en abondance, ou à l'issue d'un travail de groupe (agricole ou de construction), qui s'achève toujours par le partage d'un pot de bière. Certaines aînées cherchent à organiser de tels travaux, car l'ivresse qu'ils occasionnent alors est valorisée : elle favorise l'émission de chants qui véhiculent des valeurs relatives au statut des femmes.

Enfin, l'ivresse des jeunes enfants est rare. En temps ordinaire, ils ont peu accès à la bière de sorgho. Ils ne boivent le plus souvent que ce que leurs aînés leur font boire en partageant avec eux leurs calebasses de bière ou profitent des éventuels surplus de boisson en période cérémonielle.

\section{Une boisson cérémonielle}

Plusieurs termes utilisés pour désigner la bière de sorgho laissent entendre que ce breuvage a une certaine affinité avec l'invisible, et qu'il sied aux cérémonies rituelles et religieuses. Le terme sulum est souvent spontanément explicité comme étant la contraction de l'expression «siu lim », l'eau de «siu». Le siu est une entité protectrice, présente le plus souvent sous la forme d'un gros arbre à l'extérieur des maisons. Des sacrifices réguliers, inaugurés par un versement de bière sur l'arbre, sont supposés entretenir la vigilance de cette entité face aux attaques sorcières. L'exactitude de cette étymologie n'est pas attestée, mais sa mention régulière atteste d'une bonne diffusion de cette idée. Deuxièmement, la bière est désignée par l'expression « eleeu lim », ce

7 Être saoul se dit «sulum kuw ». Le terme sulum (qui est couramment utilisé pour désigner la bière mais s'applique également à toute boisson) est ici associé au verbe « tuer ». " Je suis saoul » se dit ainsi littéralement « la boisson me tue » (sulum kuw-m). D'autres expressions construites à partir du verbe tuer disent aussi un état d'ébranlement. Par exemple, «nyosi kuw-m » signifie « j'ai faim », litt. « la faim me tue ».

8 Contrairement à la bière de sorgho, son usage, fréquent, n'est pas rituel mais festif et médicinal. 
qui signifie « l'eau du génie de brousse ». Cette formule suggère que la transformation du sorgho en bière a quelque chose de mystérieux, qui échappe aux humains. Certains interlocuteurs pensent que ce sont les génies de brousse qui ont appris aux hommes comment fabriquer la bière. Auxiliaires des devins et des sorciers, ces génies seraient porteurs d'un savoir qu'ils transmettent aux hommes ayant réussi à les apprivoiser.

Par ailleurs, la fabrication de la bière de sorgho est un support pour penser le comput des rites annuels. La description d'une cérémonie commence souvent en indiquant la date à laquelle les femmes mettent le sorgho à germer pour la préparation de la bière $^{9}$. Mais plus encore, la fabrication de la bière est déjà le rite lui-même. Dès que le sorgho est mis à germer, des interdits relatifs au rituel concerné peuvent déjà devoir être respectés. Il arrive qu'une personne faisant l'objet d'un rite individuel soit déjà affectée par la procédure rituelle dès le moment du pilage du sorgho germé qui servira à fabriquer la bière.

L'utilisation de la bière de sorgho persiste dans les rites initiatiques et funéraires adaptés aux contraintes chrétiennes ${ }^{10}$. Lorsque le bénéficiaire de ces cérémonies (initiant ou défunt) est chrétien, il peut faire l'objet d'une cérémonie à l'église, mais des libations de bière et des mises à mort animales sont également effectuées dans le cadre domestique, comme cela est prescrit dans les cérémonies non-chrétiennes. Elles ne se font pas, alors, sur un autel dédié aux ancêtres ou au défunt, mais dans la cour intérieure de la maison, à même le sol. De la bière est toujours offerte aux participants, car offrir cette boisson est un élément fondamental de l'hospitalité kabyè. Ajoutons que l'interdit de consommer de l'alcool édicté par les Assemblées de Dieu est souvent difficilement respecté en contexte rural, car cela revient à s'extraire d'importants cadres de sociabilité. Comme dans un cas constaté en 2007, une fervente prosélyte ne manquera pas de fabriquer la bière de sorgho nécessaire à l'initiation de ses fils ${ }^{11}$.

Boire de la bière en grande quantité peut faire atteindre un état d'ivresse qui est recherché pour la participation à certaines danses rituelles nécessitant que les danseurs soient particulièrement démonstratifs. C'est le cas pour la danse qui inaugure les funérailles, qui est animée par des groupes de danseurs amenés par les gendres de la personne décédée. Les gendres prennent soin de fournir suffisamment à boire aux personnes qu'ils invitent à danser pour les représenter. C'est aussi le cas lors des danses kaming, organisées lors des rites des prémices des ignames « femelles ${ }^{12}$ », où les hommes dansent avec arcs et carquois. Ces dernières attirent désormais peu de

9 E. Jolly (2004 : 172-175) rend compte d'un usage similaire chez les Dogon, où la préparation de la bière sert d'articulation entre plusieurs rites successifs : «A travers ses phases de fabrication, de maturation et de consommation, la bière introduit de l'ordre - c'est-à-dire du sens et de la continuité - à l'intérieur des rituels ou entre les rituels ». J.-F. Vincent (1991 : 338) parle également d'un « compte à rebours de la bière » chez les Mofu du Cameroun.

10 Les personnes séduites par une confession monothéiste (20 à 30\% dans ma zone d'enquête) se tournent très majoritairement vers le christianisme : principalement le catholicisme, mais aussi le protestantisme et les églises évangéliques. Les Kabyè musulmans sont rares.

11 C. Piot (2010 : 108-110) a bien montré que les pratiques chrétiennes introduites via les Assemblées de dieu se font dans la coexistence avec les «traditions ». Comme il l'indique, il court de nombreuses histoires portant sur des pasteurs prêchant le respect d'interdits chrétiens, mais ne respectant pas euxmêmes ces préceptes. La consommation de la bière en fait partie.

12 Les tubercules de cette espèce sont de plus petite taille que ceux dits « mâles ». 
monde. Les jeunes ont « honte » d'y participer dans un accoutrement qu'ils qualifient d'archaïque. Or, lorsque des danses kaming « de fête » (c'est-à-dire extraites d'un cadre cérémoniel) sont organisées par la paroisse catholique, une foule importante s'y rend. Une des principales explications à cet intérêt est que la bière y est offerte : "Les gens vont là-bas parce qu'il y a à boire. Si tu n'as pas bu, tu as honte de danser. Tu restes trop calme », expliqua ainsi un jeune cultivateur en novembre 2006.

Désinhiber n'est pas l'effet recherché par la sur-consommation infantile de bière que nous interrogerons ici. Il est même fait peu de cas de l'ivresse à laquelle parvient l'enfant, si ce n'est pour indiquer qu'il doit s'y habituer. En quoi cette pratique peutelle alors servir le rite?

\section{Emplir un corps d'enfant}

Chaque année, environ deux mois après le début de la saison des pluies, pendant la phase finale de la croissance du petit mil, un personnage cérémoniel doit accomplir un parcours qui annonce à l'ensemble du pays kabyè l'ouverture d'une période qui est associée au renouveau des promotions initiatiques et à l'épanouissement des plantes cultivées. Pour ce faire, il lui suffit de se rendre sur le marché de certains villages et dans certaines maisons cérémonielles. Le nom donné à ce personnage insiste sur le caractère performatif de son seul passage. Il est appelé dedu : « le passant », ou le « marchant/ marcheur ». Ses déplacements sont considérés comme ayant eux-mêmes une influence positive sur la productivité agricole et la tombée de la pluie. Qu'est-ce qui leur confère une telle efficacité ? L'identité particulière de ce ritualiste, les objets puissants qu'il tient avec lui, et la consommation démesurée de bière à laquelle il est contraint de s'adonner dans les sites qu'il doit atteindre.

Ces déplacements sont d'abord évoqués par les responsables rituels comme étant accomplis par un grand prêtre traditionnel, un « cojo ». Un tel officiant est envisagé comme détenteur d'un pouvoir immanent sur les forces de la nature, à la manière des rois sacrés. Son corps, sa parole et sa pensée sont appréhendés comme étant reliés aux éléments de la nature qui s'exercent sur le territoire dont il a la charge. Une ancienne pratique voulait ainsi que lorsqu'une sécheresse menace la population, le cojo soit exposé en plein soleil jusqu'à ce que la pluie revienne (Verdier 1962 : 26). Il était mis dans la position de la terre desséchée ${ }^{13}$. Le comportement quotidien d'un grand prêtre doit en théorie être régi par la nécessité de maintenir l'équilibre des forces naturelles. De nos jours, rares sont ceux qui respectent véritablement les lourds interdits associés à ce statut, et les modifications climatiques observées sont attribuées aux défaillances des ritualistes actuels. Ne pas s'éloigner des terres de sa localité, n'être vêtu que de peaux animales, ne pas serrer la main d'une personne pour la saluer, conserver un comportement mesuré en tout temps, sont des contraintes que peu de jeunes sont prêts à accepter. Elles sont difficilement compatibles avec un travail salarié :

«Celui que 1'on veut faire cojo aura peur, peut-être parce qu'il a la jeunesse

13 Selon un cojo de la localité de Lama, à l'époque où Gnassingbe Eyadema, Kabyè lui-même, était président du Togo (entre 1967 et 2005), il lui est arrivé, lors de sécheresses prolongées, de convoquer des grands prêtres pour les faire attacher en plein soleil. En réinterprétant des pratiques relatives au pouvoir des grands prêtres sur la nature, le président se serait octroyé une position de supérieur de ce corps de spécialistes. 
(evebide) dans le sang. On lui laissera faire les cérémonies pendant un temps, et puis on verra s'il peut rester. Certains pensent que c'est honteux d'être en peaux. Il ne faut pas se promener en désordre ni traverser la rivière [qui marque une limite territoriale]. Tu ne peux pas aller boire en désordre dans les funérailles. Si tu vas dans les funérailles, tu restes à part, tout le monde sait que tu es kibalo ${ }^{14}$. Tu ne te mêles pas aux autres gens. Personne ne lui cherche des querelles, ne l'insulte ou le tape, et lui non plus, sa main ne monte pas sur quelqu'un ${ }^{15}$. »

Ces ritualistes occupent une place décisive dans l'organisation de la vie rituelle kabyè. On compte jusqu'à une dizaine de grands prêtres dans chaque localité, chacun étant appréhendé comme le descendant du fondateur mythique du village, d'un clan ou d'un groupe lignager. Responsables des cérémonies organisées pour les divinités du territoire, ils encadrent aussi les rites initiatiques masculins et féminins, qui se tiennent en partie dans les bois sacrés dédiés aux divinités agolima.

Le cojo jouant le rôle de « passant » est évoqué comme étant le grand prêtre descendant du premier couple mythique venu du ciel, dont l'union et l'installation dans la localité de Farendè, dans le massif du Lamadesi, aurait précédé le peuplement de l'ensemble des monts kabyè. L'observation des trajets accomplis mène au constat que le personnage qui fait ces déplacements n'est pas le prêtre lui-même, mais un enfant ou un adolescent, encore non-initié ${ }^{16}$, qui appartient au patrilignage du grand prêtre. Cet enfant est susceptible de devenir lui-même cojo à l'âge adulte. Pendant le parcours rituel, il est muni des objets qui sont les insignes du grand prêtre : un petit balai, formé par un assemblage de nervures de feuilles de palmier, qu'il tient dans sa main gauche, un bâton ressemblant à un manche de houe, qu'il appuie sur son épaule droite, et une calotte de couleur rouge. L'enfant se trouve ainsi transformé en cojo " modèle réduit ». Il peut être accompagné, lors de ses déplacements, par un homme adulte de la même maison, un frère classificatoire, ou même par le cojo en titre.

Le remplacement d'un grand prêtre par un enfant incite d'abord à considérer que, plus que les déplacements du grand prêtre lui-même, c'est la circulation des objets emblématiques de cette charge qui importe. De fait, il s'agit de mettre en service ces attributs du cojo. A partir de ce jour, et pendant toute la période de la saison pluvieuse où prennent place les initiations masculines et féminine, le balai, le bâton et la calotte rouge doivent rester « sortis », appuyés contre le mur de la chambre du prêtre. Cela en dehors des moments où ils sont portés par l'enfant.

Le balai et le bâton sont présentés comme étant en contact avec l'espace céleste, associé au dieu suprême, èso, qui détient les ressources dont les hommes ont besoin sur terre (notamment la pluie et les semences). Cela transparaît dans la façon dont est décrit le rôle joué autrefois par le balai dans l'intronisation d'un nouveau cojo ${ }^{17}$. L'impétrant était saisi au moment de son accession au dernier grade initiatique et conduit jusque dans un ègolimiyè, évanoui après la mise en contact avec un produit qui permettait à son âme d'effectuer un voyage céleste. Son corps restait étendu dans une case, jusqu'à ce

14 Autre mot pour désigner un grand prêtre.

15 Doyen du lignage de Manjadè, 06/2010.

16 Garçons et filles ne débutent leur initiation que vers dix-huit à vingt ans.

17 Les rares descriptions recueillies à ce sujet sont dues à deux grands prêtres des localités de Soumdina et Lama. Elles rejoignent la présentation qu'en fait R. Verdier (1982: 27). 
que le balai, envoyé par èso, « descende du ciel », et sanctionne définitivement le choix du nouveau prêtre. Qu'il touche le corps de l'initiant à la poitrine, et celui-ci mourait ; que le nouveau cojo l'évite ou l'attrape (Verdier 1982:27), et cela confirmait le choix des hommes. Selon R. Verdier (ibid.), cet objet symbolise « la parole de Dieu » (èso tom), et « la puissance (la force) de Dieu » (èso dong). Quant au bâton que tient le cojo, il est parfois présenté par ceux qui le détiennent comme une monture leur permettant d'accéder au ciel pour converser directement avec èso.

L'exposition de ces objets à l'air libre et leur circulation sur le territoire où s'exerce leur influence contribueraient au maintien d'un régime de pluies soutenu, mais aussi à la maturation des plantes cultivées et des initiants. L'efficacité attribuée à ces objets est bien affirmée dans ce passage d'une prière prononcée dans l'une des maisons visitées par l'enfant-cojo : «Vos balais, depuis le jour où j'ai sorti vos balais, je n'ai pas vu la pluie. Qu'est-ce qui s'est passé ? », demande-t-on aux grands prêtres décédés. Les parcours de l'enfant sont considérés comme réussis lorsqu'ils ont lieu sous une pluie battante. En 2010, le « passage » de l'enfant a été retardé pour un motif qui atteste de la croyance en l'efficacité de ces cérémonies. Les pluies modérées de début de saison pluvieuse étaient tombées en retard, et les plants n'étaient pas suffisamment hauts et solides pour supporter, sans être endommagés, les fortes pluies qui étaient attendues dès après le passage de l'enfant-cojo.

Le silence doit être respecté au passage du garçon. Les personnes qui le croisent ne peuvent le saluer que par des $\operatorname{clics}^{18}$. Les cultivateurs qui travaillent à la houe doivent cesser leurs mouvements. Les femmes qui sont en train d'utiliser une meule de pierre pour leurs préparations culinaires doivent s'arrêter. Un moulin à moteur qui se trouverait près du passage du cojo doit également être arrêté. Le non respect du silence impliquerait que les accompagnateurs de l'enfant saisissent une volaille pour la sacrifier sur le balai et le bâton du cojo, afin de réparer l'offense commise. D'après les officiants du clan Kumberi, auquel appartient l'enfant-cojo, ne pas respecter ce silence risquerait de " gâter le pays et la nourriture »; la pluie pourrait ne pas tomber, et « la mort » se répandrait. C'est en particulier le mûrissement final des grains du petit mil qui serait mis en danger. Comme en d'autres sociétés de l'aire culturelle voltaïque, le moment où le grain est bien formé et entame sa phase finale de croissance est une période critique. On dit alors que le mil est " plein/enceint » (mila haa en kabyè). Chez les Kasena du Burkina Faso, une période de silence rituel doit pareillement être respectée pendant l'intervalle de temps où le mil, pas encore tout à fait prêt à être récolté, " accouche » ses grains. Tout dérangement bruyant pourrait faire surgir un vent qui ferait « avorter » le mil (Liberski-Bagnoud 2002 : 176).

Les déplacements rituels de l'enfant-cojo donnent lieu à une séquence qui consiste à lui faire boire une grande quantité de bière de sorgho. Une fois parvenu au marché ou dans l'une des maisons à visiter, l'enfant est assis sur une pierre réservée au grand prêtre. Si l'on se trouve au marché, il attend là pendant que ses accompagnateurs vont puiser de la bière auprès de l'ensemble des vendeuses de cette boisson. Le pot dans lequel la bière a été récoltée est ensuite déposé devant l'enfant, à côté de la grande calebasse utilisée habituellement par le grand prêtre pour transporter les substances libatoires. 
Cette calebasse est ensuite emplie de bière et sera donnée à boire à l'enfant. La bière qu'il consomme constitue ainsi un échantillon de l'ensemble de la bière produite ce jour-là dans la localité concernée. Lorsque le trajet accompli s’achève dans une maison, la boisson consommée aura été préparée dans la maison visitée.

Ces lieux dans lesquels l'enfant doit boire sont significatifs. Le marché représente un condensé du territoire. Il est subdivisé en plusieurs cercles de pierres qui sont associés aux subdivisions territoriales de la localité, et sur lesquels s'installent les vendeurs. Cet espace, placé sous la juridiction d'une divinité ègolimiyè, est un lieu de recours pour la mise en œuvre de cérémonies qui concernent l'ensemble du territoire d'une localité. Quant aux maisons visitées, elles correspondent à différents sites d'essaimage de la population. Certaines d'entre elles se situent sur les limites est et ouest du territoire sur lequel le grand prêtre représenté par l'enfant exerce son influence. Il s'agit donc de boire dans des lieux qui symbolisent l'aire d'action du cojo, et d'ingérer une bière qui a été produite par les différentes composantes claniques et lignagères qui dépendent de ce prêtre.

Que l'on se trouve au marché ou dans une maison, la séquence d'ingestion de bière par l'enfant repose sur des codes similaires. Environ cinq à six personnes forment une file et se succèdent face à l'enfant pour lui faire boire des calebasses de bière de sorgho. Lors d'une séquence observée sur un marché, c'est d'abord la femme du grand prêtre en titre, puis plusieurs hommes appartenant au patrilignage du grand prêtre qui se sont relayés devant l'enfant pour le faire boire. Dans une maison, ce sont les femmes, puis les hommes de la maison visitée qui ont fait boire l'enfant.

Sur le marché, le contenu de chaque calebasse de bière est partagé entre l'enfant et la personne qui le fait boire. En gardant les genoux légèrement fléchis, la femme saisit à deux mains la grande calebasse, la porte à ses lèvres pour avaler une petite gorgée de bière, puis approche la calebasse de la bouche de l'enfant, pour lui faire boire quelques gorgées ${ }^{19}$. Elle ramène ensuite la calebasse vers sa bouche et boit à son tour, en posant ses lèvres sur le bord de calebasse opposé à celui par lequel l'enfant avait bu. Le récipient se trouve alors presque vidé. La femme le repose sur le sol, et salue l'enfant par des clics. La calebasse est de nouveau emplie, et la personne suivante fait boire l'enfant de la même façon, puis transmet à son tour la calebasse à celui qui la suit. A aucun moment l'enfant ne prend lui-même cet objet dans ses mains.

Dans une maison où j'ai pu observer cette pratique, la commensalité entre l'enfant et les participants au rite se fait un peu différemment. Dans un premier temps, l'enfant et ses accompagnateurs, mais aussi tous les adultes de la maison visitée, boivent librement une première calebasse de bière, distribuée en signe d'accueil. On prend soin d'attendre que tous aient terminé de boire avant d'ouvrir la séquence principale consistant à faire boire l'enfant. Ce sont d'abord les femmes de la maison, puis les hommes, qui viennent s'accroupir les uns après les autres devant le cojo, le saluer par des clics, et lui donner à boire. La quantité de boisson que l'enfant doit alors lui-même absorber est supérieure à ce qu'il boit sur le marché. Après que la personne qui le fait boire a prélevé une petite gorgée de bière dans la calebasse, c'est l'enfant qui boit seul l'intégralité de chaque

19 Mouiller ses lèvres dans la calebasse de bière que l'on veut tendre à la personne à qui l'on donne à boire, est la manière commune et polie d'offrir à boire à quelqu'un. 
calebasse de bière. Le garçon semble alors réduit à l'état de contenant. En dehors de l'acte de déglutition, son corps est dans une position de grande passivité. Son ventre se gonfle progressivement et devient douloureux. Étant donné le rythme auquel il est tenu de boire, l'enfant est vite gagné par l'ivresse. Indisposé, il cherche à changer de position et à s'allonger avant d'avoir fini de boire la totalité du liquide qu'il est supposé ingurgiter. Les hommes qui l'entourent l'en dissuadent en lui disant qu'il reste encore très peu à boire et qu'il pourra se reposer ensuite.

A Farendè, d'autres rituels nécessitent que les participants au rite viennent faire boire le cojo un à un : non pas l'enfant, mais bien le cojo adulte en titre. Là encore, ce dernier n'intervient dans le rite que par sa seule présence buvante. Son rôle se résume à être assis sur sa pierre et à avaler. En imposant ce geste de "donner à boire au cojo ", le rite souligne les liens de dépendance et/ou d'appartenance lignagère qui lient ce personnage à chaque membre adulte des lignages qui bénéficient des cérémonies. Mais lorsque ce type de cérémonie est pratiqué avec un enfant qui se substitue au grand prêtre, on se rend compte qu'il ne s'agit pas seulement, alors, de nouer des liens par un boire ensemble. Il s'agit tout autant d'emplir ce personnage de boisson, de manière à produire l'image saisissante d'un corps à emplir, tel une outre.

La séquence s'achève par une prière du doyen de la maison où l'on se trouve. Il la prononce accroupi devant l'enfant-cojo en tenant dans ses mains la calebasse de l'enfant, qui ne contient plus que la lie. Il s'adresse alors aux grands prêtres décédés. Il leur demande que la pluie tombe abondamment, et que le vent (emblématique de la saison sèche) ne souffle pas. La prière s'achève par le versement de la lie sur le sol, aux pieds de l'enfant. Celui-ci apparaît à la fois comme l'objet et le destinataire du rite. C'est son corps que l'on emplit au-delà de la soif, c'est face à lui que se tient le doyen lorsqu'il énonce la prière qui clôture le rite, et c'est entre ses pieds qu'est accompli le geste conclusif de versement de la lie. Représente-t-il ici uniquement le grand prêtre qu'il remplace ou les grands prêtres disparus auxquels on s'adresse dans la prière ? Il semble qu'il soit ici avant tout perçu comme le véhicule de la prêtrise, « cojotu ».

\section{Pourquoi un enfant?}

Il peut sembler paradoxal que la tâche d'accomplir ces parcours silencieux et de porter les emblèmes du cojo soit confiée à un enfant. Le statut d'enfant n'est pas particulièrement valorisé. Une personne n'a aucun poids décisionnel tant qu'elle est désignée comme «enfant ", c'est-à-dire avant d'avoir achevé son initiation. Dire de quelqu'un qu'il est enfant, c'est dire qu'il ne peut émettre publiquement une opinion engageant sa maison ou son patrilignage. Sa capacité de jugement n'est pas considérée comme valable.

Les responsables rituels interrogés à ce sujet commencent par expliquer la présence de cet enfant par son caractère formateur : " Tu dois lui montrer comment faire les cérémonies. Quand tu ne seras plus là [décédé], il pourra te remplacer et faire les cérémonies à ta place. Si on ne lui apprend pas, comment deviendra-t-il $\operatorname{cojo}^{20}$ ? ».

Toutefois, la présence de l'enfant n'est pas requise pour l'ensemble des cérémonies 
conduites par un grand prêtre. Pourquoi cet apprentissage se restreindrait-il à une partie seulement des activités de cojo ? L'argument didactique n'est pas suffisant. Une autre raison pratique est évoquée : les trajets à accomplir seraient trop fatigants pour le grand prêtre en titre, qui est un homme vieillissant. " L'enfant est plus dynamique, il peut aller partout sans se fatiguer. ». Or, l'observation des cérémonies vient indiquer que la présence d'un enfant n'est pas un pis-aller. Elle est inscrite dans la trame du rite, et la position d'enfant-cojo est un statut à part entière. C'est l'absence de l'enfant qui est problématique. En 2010, l'adolescent qui était censé participer à un de ces parcours rituels n'était pas là au moment prévu. On est allé le chercher chez lui mais il est resté introuvable. C'est le grand prêtre en titre, contrarié, qui a dû se résoudre à occuper la place de cojo. Il a réprimandé publiquement l'adolescent quand ce dernier l'a finalement rejoint, et lui a remis aussitôt les attributs de la prêtrise.

Le frère du grand prêtre en titre, proviseur à la retraite, expliqua la présence de l'enfant en ces termes :
« J'ai moi-même fait cela lorsque j'étais un petit enfant. C'est comme cela que l'on apprend à l'enfant comment faire les cérémonies, quels sont les chemins à suivre. L'enfant on lui donne les outils de cojo parce qu'il est pur, il n'a pas encore eu de rapports avec une femme. Quand tu grandis, que tu as l'âge d'être èvalo ${ }^{21}$, on te remplace. »

Le terme «pur» utilisé par les francophones pour expliquer ce recours à un enfant, correspond au terme kabyè " ciri », ce qui signifie d'abord " propre », mais aussi « tout », « complètement» (Marmor 1999). Dire de quelqu'un qu'il est ciri, c'est donc insister sur le fait qu'il est « intact», complet. C'est le fait de ne pas encore avoir eu de rapports sexuels qui confère cet état aux enfants. Le garçon qui revêt les atours du cojo occupe cette fonction de substitut pendant plusieurs années. Ce n'est que lorsqu'il est susceptible d'avoir des rapports sexuels avec une femme qu'il ne peut plus jouer ce rôle rituel. Il deviendrait alors plus « faible » (èjam) qu'un jeune enfant.

Pour les garçons comme pour les filles, l'entretien de rapports sexuels est en effet envisagé comme vecteur d'une perte d'intégrité. Pour un homme, avoir des rapports sexuels avec une femme est perçu comme un facteur d'amoindrissement de ses propres forces, car cela implique une perte de sperme/sang (le même mot, calim, désigne ces deux substances corporelles). C'est aussi une mise en contact potentielle avec une forme de pollution féminine : le sang menstruel. Ce n'est donc pas un savoir-faire particulier qui confère à l'enfant une compétence lui permettant d'occuper une place centrale dans cette pratique rituelle - les informateurs y insistent : « il ne connaît rien, il faut tout lui montrer »- mais une qualité corporelle. L'intégrité de son corps (pas encore pollué ou entamé par l'acte sexuel avec le sexe opposé) rend l'enfant apte à figurer un parfait contenant, et à travers lui, l'image d'une plénitude et d'une abondance potentielle.

C. Piot a montré que chez les Kabyè, comme chez les Dogon et les Bambara du Mali, les enfants sont associés à une androgynie symbolique qui leur confère une certaine complétude sexuée :

" If Kabre children are sexually ambiguous and, as it were, symbolically complete, they must nevertheless become sexually single and 'incomplete' as they mature.

21 Premier grade initiatique masculin. 
The task of creating incomplete, gendered persons - of deconstituting androgynous persons — is the aim of a child's initiation ceremonies » (1999: 79).

L'androgynie apparaît par ailleurs, dans un mythe rapporté par R. Verdier (1982 : 25), comme caractéristique du premier homme venu du ciel. Les organes féminins se trouvaient sous ses aisselles. L'enfant, symboliquement androgyne, semble bien placé pour représenter au mieux un grand prêtre qui, lors de son intronisation, est fabriqué à l'image du premier homme.

Chez les Dogon, qui sont comme les Kabyè une population de langue voltaïque, le prépuce témoigne de la féminité du garçon, et le clitoris représente la part masculine de la petite fille (Dieterlen 1973 : 222). L'état d'androgynie perdure jusqu'à la circoncision ou l'excision. Avant cette opération, l'enfant intact est dit «purolu », « non impur » (Liberski 1989 : 59). D'après G. Dieterlen (1941 : 208), le qualificatif de purolu est également utilisé pour désigner les enfants, « tant qu'ils n'ont pas eu de rapports sexuels ». Le circoncis est qualifié de «puru ». Ce terme, traduit par « impur », connote l'idée d'une perte de vitalité, de vide, d'incomplétude, et peut aussi renvoyer à l'idée de saleté (Liberski 1989 : 42-43). Il s'oppose à la notion d'oms, « vivant », traduit communément par « pur ». L'opposition sms/puru repose sur « une idée du « vivant» comme corps lisse, bouché, voué à la complémentarité gémellaire, mais aussi en quelque sorte défini par l'espace du village » (ibid. : 60).

Chez les Kabyè, la différentiation sexuée à laquelle procèdent les initiations masculine et féminine ne passe pas par la circoncision ou l'excision. Mais la vérification de la virginité par diverses entités est quelque chose de récurrent, à plusieurs grades de l'initiation masculine. Elle est centrale dans l'initiation féminine. En théorie, l'accès à la sexualité n'est autorisé qu'après la fin de l'initiation. Le statut d'enfant (non-initié) est donc fortement associé à l'absence de sexualité et à l'intégrité que cela confère. Les représentations dogon relatives à la sexuation de la personne peuvent donc nous guider pour comprendre ce qui peut motiver l'usage d'un corps d'enfant comme contenant dans le rite kabyè qui nous intéresse ici. Chez les deux sociétés, le corps de l'enfant tient son caractère " complet » du fait que son genre n'a pas encore été construit et définitivement fixé par l'initiation, ni défini par l'activité sexuelle. Porteur simultanément du féminin et du masculin, on a recours à ce corps intact parce qu'il représente un potentiel de vie. Il convient d'emplir ce corps « lisse » de bière de sorgho, de manière à créer une image du « plein », associée à l'abondance et au vivant ${ }^{22}$.

\section{Emplir des contenants anthropomorphes}

Deux autres pratiques rituelles nécessitent de produire l'image d'un corps plein, à l'aide de bière de sorgho ou de matières dérivées de la fabrication de la bière. Elles ont lieu dans les localités de Lama et Soumdina, au sein du massif montagneux le plus vaste et le plus peuplé, qui était à l'époque précoloniale dans un rapport antagoniste avec le massif originel du Lamadesi $^{23}$. Il ne s'agit pas alors d'emplir un corps humain de bière, mais un réceptacle dont

22 Nous retrouvons ici une conception du corps humain comme récipient qui est comparable à ce que J.P. Warnier (2009) a mis en évidence chez les Mankon du Cameroun, où le corps du roi apparaît comme une « tirelire vitale ».

23 Les habitants des deux massifs étaient ennemis. Les deux massifs se distinguent par des différences dialectales et rituelles remarquables (plus appuyées que celles constatées au sein d'un même massif). 
certaines parties sont présentées comme évoquant un corps de divinité ègolimiyè, d'aspect humain. Tant par leur forme que par leur finalité, ces pratiques peuvent être vues comme prenant le relais du rituel initial impliquant d'emplir de bière le ventre d'un enfant.

\section{Couples de pots}

Quelques semaines après les parcours rituels de l'enfant-cojo, un à deux jours avant les sorties initiatiques collectives - phases capitales dans la production de nouveaux hommes et femmes adultes - des pots dits sèyisi sont acheminés dans des bosquets où siègent des divinités agolima. Sont appelés agolima, les fondateurs mythiques du village et de certains lignages (le couple issu du ciel et leurs enfants), mais aussi des puissances du sol auxquelles ces premiers hommes ont commencé à offrir des sacrifices pour que leur soit concédée la possibilité d'habiter l'espace terrestre. Ces deux catégories d'agolima sont pareillement vues comme ayant la capacité de se rendre auprès du dieu-ciel èso pour ramener aux hommes ce dont ils ont besoin pour vivre sur terre. Chaque ègolimiyè est compétent en un ou plusieurs domaines (la pluie, les cultures, la chasse, etc.), et exerce son influence sur une portion de terre déterminée ${ }^{24}$.

Les pots sèyisi les plus anciens, désormais rares, sont surmontés d'une partie où l'on peut reconnaître une tête et un buste de forme humaine. Ils représentent les ancêtres descendus du ciel et leurs enfants. Ceux de conception moderne ont la forme d'une grosse poire. A l'image des ancêtres fondateurs, ces pots sont sexués (mâle et femelle) et vont par deux. Ce sont donc des représentations d'entités aptes à communiquer avec èso (Figure 1).

Au moment de leur dépôt dans les agolima, les sèyisi sont emplis de bière de sorgho provenant du ou des lignages liés à l'ègolimiyè dans lequel on se trouve. Le jour-même ou le lendemain de l'emplissage des pots, le prêtre responsable du site y puise de la bière et en asperge des pierres et troncs d'arbres dispersés dans le bosquet. Lui-même et les autres officiants présents (grands prêtres ou représentants des groupes lignagers qui lui sont liés) boivent ensuite. A Lama, le mode d'adresse aux agolima est particulier. Dans chaque ègolimiyè visité, les versements de bière sont étroitement coordonnés à l'appel musical d'une vingtaine d'agolima implantés sur le territoire de Lama ou sur des localités voisines. Tous sont liés aux groupes lignagers de Lama. Cette interpellation, émise avec une flûte en bois et un cor en corne, commence par l'injonction : «Appelez la pluie pour qu'elle descende ! Viens, ton enfant meurt ! » Puis chaque ègolimiyè est appelé par une phrase respectant la structure suivante : "Kibiniu kicuwluw [nom] viens yeee, viens, ton enfant meurt! 》

On les incite ainsi à faire tomber la pluie, indispensable à la vie sur terre, et à veiller sur les jeunes gens que l'on s'apprête à initier. L'expression « ton enfant meurt » fait référence aux initiants qui sont sur le point d'être mis en contact avec plusieurs des divinités auxquelles les grands prêtres s'adressent. Annoncer la mort d'une nouvelle génération, c'est annoncer le risque que la société cesse de se perpétuer. Ces invocations se poursuivent les jours suivants, en préambule des sorties collectives des initiants des deux sexes, qui se réalisent de préférence sous la pluie.

24 Les caractéristiques et le mode de fonctionnement attribué à ces entités permettent de les rapprocher des puissances du territoire nommées « peau de la terre », décrites en plusieurs sociétés de l'aire culturelle voltaïque (Fortes 1945 ; Goody 1956 ; Liberski-Bagnoud 2002). 


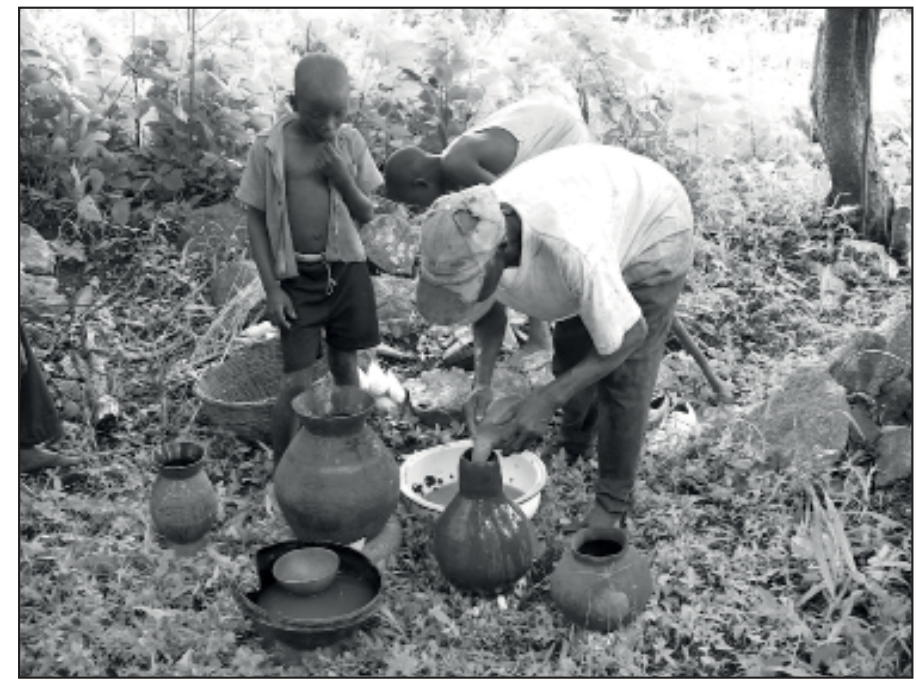

Figure 1 : Emplissage d'un pot anthropomorphe avec de la bière issue de plusieurs lignages (C) Marie Daugey, Soumdina 2010

Les sèyisi restent ensuite vides pendant environ un mois et demi dans les agolima, jusqu'au jour appelé yoyoka, qui annonce l'entrée dans une période de transition amenant à la saison sèche. Ce jour-là, les pots sont remplis de bière de sorgho et de nouvelles libations sont effectuées. A Lama, les agolima sont appelés de nouveau en musique et incités à « retourner dans leurs maisons ». Avant de verser la lie, le grand prêtre qui détient plus que les autres un pouvoir sur la pluie prononce une prière après laquelle une grande averse de pluie est censée tomber. La compétence de ce prêtre est jaugée par les plus âgés au regard de l'intensité de cette averse. Comme l'indiqua Kijanday Begedu, chargé d'accomplir cette prière jusqu'en 2009, dans sa prière, l'officiant « demande à èso la pluie et le soleil, sompiye et poya ${ }^{25}$ ». Sompiye, qualifié de mâle, est un fruit sauvage ; poya, féminin, désigne un cauri. Le premier représente le soleil et le second, la pluie. L'association de ces deux principes est envisagée comme propice à la procréation, comme le souligne le fait que sompiye et poya ornent des boucles d'oreilles censées favoriser la conception des enfants. Autrefois, c'est d'ailleurs à cette période de l'année que les enfants étaient conçus de manière préférentielle.

Les libations accomplies à l'aide des pots sèyisi consistent ainsi à présenter aux divinités l'image d'elles-mêmes comme corps-contenants réunissant masculinité et féminité et figurant un potentiel générateur. Leurs remplissages successifs offrent l'image de ce que l'on désire qu'elles soient : une réserve de pluie et de ressources favorisant le déploiement de la vie ${ }^{26}$.

25 Avant yoyoka, il demande uniquement une pluie abondante dans ses prières.

26 Chez les Dogon, la bière de sorgho représente la fécondité et un vecteur de vie (Jolly 2004 : 218). Les pratiques de la bière décrites ici rejoignent implicitement cette même idée. 


\section{Ventres irrassasiables}

Enfin, trois mois après yoyoka, en début de saison sèche, d'autres cérémonies impliquent d'emplir un réceptacle évoquant un corps humain. Elles se déroulent auprès d'agolima compétents dans le domaine de la satiété alimentaire. La maturation finale du sorgho et la capacité de son grain à rassasier les hommes sont alors en jeu. Dernière nourriture à être récoltée au cours d'une année, le sorgho a longtemps été la principale céréale fournissant la farine permettant de préparer le plat de base de l'alimentation des Kabyè : la «pâte » $(m u t u)$, toujours agrémentée d'une sauce ${ }^{27}$.

Un contenant nommé cèluw se trouve dans les sites dédiés aux agolima concernés. Le mot cèluw désigne une grande assiette en argile qui est tellement large qu'on la remplit difficilement. Il désigne ici de manière métaphorique un ventre ou un estomac qui n'est jamais complètement plein et rassasié. Le réceptacle cèluw correspond à un pot d'argile simplement enterré ou intégré à une forme maçonnée parfois présentée comme anthropomorphe et sexuée, sur laquelle est repérée une " tête », et dont l'ouverture est désignée comme la « bouche ». «C'est la forme d'un homme qui est là-bas. S'il est rassasié, ses enfants n'auront plus faim », indique en décembre 2007 l'un des doyens de lignage de Soumdina, infirmier, qui prenait part à l'ouverture d'une telle structure.

Après l'ouverture de ce contenant, les représentants des groupes lignagers participants vérifient le degré d'humidité à l'intérieur. Si les éléments qui y avaient été déposés l'année précédente sont trop secs, c'est interprété comme le signe de difficultés alimentaires dans l'année à venir. Si une humidité subsiste, c'est que l'année qui vient sera propice. Après cette inspection, le contenant doit être comblé par un ensemble de substances variable d'un cas à l'autre, mais qui comporte toujours un liquide rougeâtre composé d'eau mêlée de drêches (tèètu) résultant de la préparation de la bière qui est consommée ce jour-là (Figure 2). Dans un cas, de l'eau enfarinée, composée d'un mélange de farine obtenue avec du sorgho vermoulu de l'année précédente et de farine issue de la nouvelle récolte est versée. Dans un autre cas, les restes du repas sacrificiel faisant suite à l'ouverture de cèluw sont déposés dans le trou avant que celui-ci ne soit empli d'eau de drêches jusqu'au trop-plein. Comme dans les situations étudiées précédemment, il convient de donner à voir un contenant qui déborde, mais qui déborde cette fois d'un ersatz de bière évoquant la capacité nutritive de cette boisson ${ }^{28}$.

L'instant du débordement donne lieu à des cris de satisfaction : « elle est pleine/ rassasiée ! ». Cèluw figure ainsi un estomac générique qu'il faut combler pour que la population puisse ressentir facilement le rassasiement. Lorsque le rite nécessite un sacrifice animal, les officiants expliquent à l'ègolimiyè dans leur prière que les « enfants » souffrent de famine et que la farine est inconsistante (" cela devient le vent »).

27 Le maïs supplante désormais souvent le sorgho et le petit mil sur le plan cultural et alimentaire. Considéré comme étant d'un meilleur rendement, il est plus apprécié par les jeunes. Ils voient la pâte « blanche » issue de sa farine comme plus « moderne » que la pâte de sorgho, « rouge ».

28 La couleur « rouge » du liquide obtenu est comparable à celle de la bière, ordinairement appelée « eau rouge ». E. Jolly (2004 : 275) souligne que chez les Dogon, la " drêche est employée comme un ersatz de bière tant qu'elle conserve un peu d'humidité (...). En suçant ce dépôt ou en consommant son jus d'imprégnation, les femmes, les enfants ou les jeunes réaffirment leur appartenance à la communauté lignagère, même si la beuverie principale a eu lieu plusieurs jours auparavant. » 
«Lorsque la femme prépare à manger, il faut qu'elle obtienne une grande quantité de pâte avec peu de farine, et qu'il y ait des restes ${ }^{29} »$.

Ainsi le rite produit-il l'image paradoxale d'une plénitude suggérant l'abondance et le rassasiement à l'aide de dérivés de sorgho (drêches, farine) qui sont des restes et symbolisent l'épuisement des vivres. Leur dilution dans l'eau permet d'opérer une transformation de ces résidus de l'état de ressource épuisée à celui de symbole de l'abondance à venir. L'usage d'un mélange d'ancienne et de nouvelle farine traduit ce souhait de voir convertir l'état des ressources alimentaires : du manque au plein.

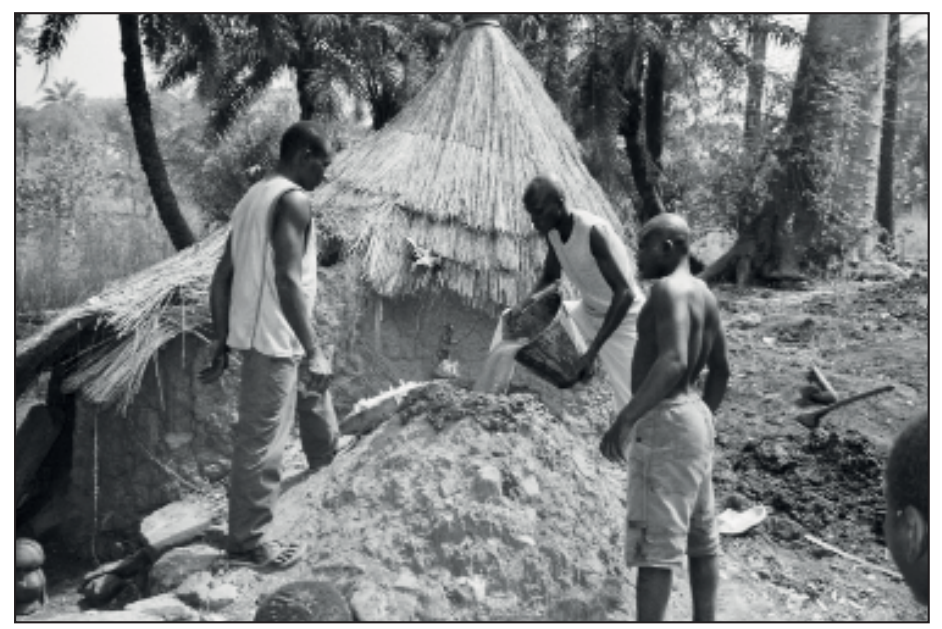

Figure 2 : La cavité cèluw, emplie de restes alimentaires - C Marie Daugey, Lama, 12 décembre 2006

\section{Conclusion}

L'ivresse inévitable à laquelle parvient l'enfant-cojo lors des rites d'inauguration des initiations et d'accompagnement du mûrissement du petit mil n'est qu'un effet secondaire du rite. Emplir de bière l'estomac de l'enfant permet de rendre visible la qualité que l'on recherche chez lui : sa corporéité. Le versement de bière dans l'enfant procède à la réification de ce dernier : il fait avant tout office de contenant. En contrepoint, le versement de bière dans des pots anthropomorphes et des cavitésventres contribue à première vue à une forme de personnification de ces objets : on les fait boire et manger tels des hommes. Toutefois, ces corps produits par le rite et la bière diffèrent des corps humains ordinaires : ils représentent une étanchéité et une androgynie dont se sont départis les hommes et les femmes adultes, construits comme des personnes finies. Ils évoquent le monde de l'antériorité. Dans ces rites, ce qui compte est moins l'intentionnalité humaine ou l'agentivité (Gell 1998) qui se trouverait projetée sur les formes anthropomorphes, que la mise en forme de dispositions physiques propres aux choses des dieux. Conçus comme des choses du divin parmi les hommes, ces corps-récipients sont manipulés (emplis, vidés), pour communiquer au mieux avec les puissances divines liées au monde de l'antériorité : en figurant des réservoirs d'abondance relevant du monde des dieux. 


\section{Références citées}

CRIDEL, Bernard, 1968. « Cueillette, chasse, pêche et alimentation chez les Kabrè ». Documents du CERK (Centre d'Études et de Recherches de Kara), pp. 63-70.

Dieterlen, Germaine, 1941. Les âmes des Dogons. Paris : Institut d'ethnologie.

DieTERLEN, Germaine, 1973. «L'image du corps et les composantes de la personne chez les Dogon », in Roger Bastide et Germaine Dieterlen (éds), La notion de personne en Afrique Noire, pp. 205-229. Paris : L'Harmattan.

FABre-VASSAS, Claudine, 1989. « La boisson des ethnologues », Terrain, 13, pp. 5-14.

FORTES, Meyer, 1945. The dynamics of clanship among the Tallensi. Being the first part of an analysis of the social structure of a Trans-Volta tribe. Londres: Oxford University Press.

Gell, Alfred, 1998. Art and Agency. An anthropological theory. Oxford: Oxford University Press.

Goody, Jack, 1967 [1956]. The social organisation of the LoWiili, seconde édition. Londres: Oxford University Press pour l'International African Institute.

JOLLY, Eric, 2004. Boire avec esprit. Bière de mil et société dogon. Nanterre : Société d'ethnologie.

LiBERSKI, Danouta, 1989. « Pour une géographie de la saleté », Journal des Africanistes, 59 (1-2), pp. 39-62.

Liberski-Bagnoud, Danouta, 2002. Les dieux du territoire. Penser autrement la généalogie. Paris : CNRS/ MSH Éditions.

Marmor, Thomas, 1999. Tom Kpou Kabıye-Fransıl (Dictionnaire Kabiyè-Français avec lexique françaiskabiyè et esquisse de grammaire kabiyè). Lomé : Comité de Langue Nationale Kabiyè (CLNK) et SIL-Togo.

Piot, Charles, 1999. Remotely global; Village modernity in West Africa. Chicago et Londres : The University of Chicago Press.

Piot, Charles, 2010. Nostalgia for the future. West Africa after the Cold War. Chicago et Londres : The University of Chicago Press.

Roberts, David, 2013. Parlons kabiyè. Paris : L'Harmattan.

VERDIER, Raymond, 1962. Les structures socio-religieuses des Kabré du Nord-Togo. Thèse de doctorat. Université de Paris Sorbonne.

Verdier, Raymond, 1982. Le pays Kabiyé. Cité des dieux, cité des hommes. Paris : Karthala.

VINCENT, Jeanne-Françoise, 1991. Princes montagnards du Nord-Cameroun. Les MofuDiamaré et le pouvoir politique, Paris : L'Harmattan.

WARnier, Jean-Pierre, 2009. Régner au Cameroun. Le roi-pot. Paris : Karthala. 